\title{
Qual é a melhor estratégia de prescrição do exercício aeróbio para aumentar o dispêndio energético em pacientes sedentários?
}

\author{
Which is the best strategy of aerobic exercise prescription to increase \\ energy expenditure in sedentary patients?
}

\author{
Felipe A. da Cunha
}

Rafael A. Montenegro

Paulo T. V. Farinatti*

\section{Resumo}

A importância do exercício aeróbio para controle do peso é bem-aceita na literatura, mas há dúvidas sobre a dose ótima de exercício com esse fim. A presente revisão descreve as bases metodológicas da prescrição do exercício aeróbio sob a ótica da otimização do dispêndio energético (DE) e redução de peso em pacientes sedentários. Nesse contexto, dentre as principais características da prescrição dos exercícios aeróbios destacase a intensidade. Para o estabelecimento da intensidade e estimativa do DE do exercício, tem sido recomendado o uso do percentual de consumo de oxigênio de reserva $\left(\% \mathrm{VO}_{2} \mathrm{R}\right)$, em razão de sua suposta relação de equivalência de 1:1 com o percentual de frequência cardíaca de reserva (\%FCR), em conjunto com as equações metabólicas do American College of Sports Medicine (ACSM) para cálculo da carga de trabalho. Porém, existem limitações na aplicação desse método, principalmente em virtude da hipotética relação 1:1 entre \% FCR-\% $\mathrm{VO}_{2} \mathrm{R}$ não se reproduzir em sessões contínuas de exercício de longa duração realizado com diferentes intensidades. Com isso, pode-se superestimar ou subestimar o DE. Outro aspecto é intensidade ideal de treinamento. Recentemente, tem-se recomendado exercícios de alta intensidade e curta duração para promover maiores benefícios sobre a aptidão cardiorrespiratória, composição corporal e fatores de risco cardiovasculares, em comparação com sessões de moderada intensidade e longa duração. Porém, aspectos relacionados com a adesão às rotinas de exercício colocam em dúvida essa estratégia, o que deve ser considerado na elaboração dos programas visando a redução de peso e promoção da saúde.

Descritores: Treinamento; Consumo de oxigênio; Gasto energético; Promoção da saúde; Aptidão física.

"Endereço para correspondência: Laboratório de Atividade Física e Promoção da Saúde, IEFD, UERJ. Rua São Francisco Xavier, 524, sala 8.121, Bloco F Rio de Janeiro, RJ, Brasil. CEP: 20550-013

Telefone: 5521 2334-0775 E-mail:pfarinatti@gmail.com 


\section{Abstract}

Aerobic exercise is an important complementary strategy for weight control, but there are questions about the optimal dose of exercise with this purpose. This review describes the methodological bases of aerobic exercise prescription, with emphasis on the optimization of energy expenditure (EE) and weight reduction in sedentary subjects. In this context, the study focused mainly on the intensity of aerobic exercise prescription. The percentage of oxygen uptake reserve $\left(\% \mathrm{VO}_{2} \mathrm{R}\right)$ - along with the American College of Sports Medicine to calculate training workloads -, have been used to define the exercise intensity and estimate EE, due to its hypothetical 1:1 relationship with the percentage of heart rate reserve (\%HRR). However, there are limitations in the application of this method, since studies applying continuous bouts of exercise with different intensities have reported that such hypothetical 1:1 relationship between '\%HRR-\% $\mathrm{VO}_{2} \mathrm{R}$ ' may overestimate or underestimate EE. Another issue refers to the optimal training intensity. High-intensity short-term exercises have been recommended to promote greater benefits on cardiorespiratory fitness, body composition, and cardiovascular risk factors compared to long-term moderate exercise bouts. However, aspects related to adherence to exercise routines cast doubt over the effectiveness of this strategy, which should be considered when designing exercise programs in the context of weight reduction and health promotion.

Keywords: Training; Oxygen uptake; Energy expenditure; Health promotion; Physical fitness.

\section{Introdução}

A prática regular de exercícios físicos tem sido considerada como uma importante estratégia de saúde pública e qualidade de vida, ${ }^{1}$ em virtude da associação entre aptidão cardiorrespiratória reduzida e maior risco de morbimortalidade por doenças cardiovasculares e metabólicas. ${ }^{2}$ Por outro lado, o aumento das taxas de sedentarismo ${ }^{3}$ e crescente prevalência de doenças hipocinéticas, ${ }^{4}$ especialmente a obesidade, destaca a necessidade de se investigarem estratégias eficazes de prescrição do exercício.

Nesse sentido, o treinamento aeróbio é valioso por induzir adaptações fisiológicas associadas ao aprimoramento da saúde. ${ }^{2}$ Porém, a prescrição ótima de exercício para maximizar tais adaptações em diferentes populações e necessidades permanece incerta. ${ }^{5}$ As características da prescrição dos exercícios aeróbios incluem intensidade, duração, frequência e tipo de atividade. ${ }^{1,2}$ A relação entre intensidade e duração das sessões de treinamento tem recebido atenção particular no contexto de programas de manutenção de peso, devido ao impacto relativo de diferentes combinações na aptidão cardiorrespiratória ${ }^{6} \mathrm{e}$ dispêndio energético. ${ }^{7}$

Em geral, aceita-se que muitos dos benefícios do treinamento aeróbio estão relacionados à quantidade total de trabalho empreendida. ${ }^{8}$ Isso explica o porquê de algumas recomendações tradicionais priorizarem exercícios de intensidade leve-moderada e duração de pelo menos 30-60 minutos, todos os dias da semana, assegurando estímulo de treinamento adequado (sem esforço excessivo ou desconforto desnecessário) e privilegiando a segurança dos exercícios, principalmente em indivíduos sedentários ou com baixa aptidão cardiorrespiratória. $^{8}$

Entretanto, há evidências consistentes de que exercícios de alta intensidade ou vigorosos, sob condições de treinamento isocalórico, seriam mais efetivos para aumentar a aptidão cardiorrespiratória ${ }^{6,9}$ e reduzir o percentual de gordura e massa gorda, ${ }^{7}$ em comparação com exercícios de intensidade leve a moderada. O mesmo raciocínio pode ser aplicado a outros fatores de risco cardiovascular, como colesterol total, HDL, triglicerídeos séricos, circunferência de cintura, sensibilidade à insulina e glicemia., ${ }^{79-12}$ Por outro lado, intensidades relativamente altas 
de exercício podem reduzir significativamente a aderência aos programas de treinamento. ${ }^{13}$ Logo, é importante considerar esses dois aspectos para a prescrição do exercício.

Para discutir a relação de dose-resposta do treinamento aeróbio sobre os marcadores fisiológicos mencionados, é necessário entender algumas possíveis limitações associadas aos aspectos metodológicos da prescrição dos exercícios aeróbios, dentre os quais: a) métodos utilizados para o estabelecimento da intensidade de treinamento e estimativa do dispêndio energético das sessões de exercício aeróbio (Quais são as variáveis de prescrição da intensidade de esforço? Como estimar o dispêndio energético das sessões de treinamento para uma determinada carga de trabalho?); b) limitações metodológicas associadas à prescrição da intensidade do exercício aeróbio; c) vantagens e desvantagens da opção por treinamento de intensidade alta ou moderada (afinal, qual seria a melhor estratégia para o emagrecimento?). O objetivo do presente texto é discutir as bases metodológicas da prescrição do exercício aeróbio com vistas à melhor estratégia para aumento do dispêndio energético em indivíduos sedentários, por meio de revisão da literatura sobre o tema.

\section{Recomendações gerais para prescrição do exercício aeróbio - o que se propõe fazer}

As intensidades dos exercícios aeróbios podem ser estabelecidas de acordo com as recomendações do American College of Sports Medicine (ACSM), valendo-se da frequência cardíaca máxima (FCmáx), unidades metabólicas (METs), consumo máximo de oxigênio $\left(\mathrm{VO}_{2}\right.$ máx $)$ e percepção subjetiva de esforço (PSE). ${ }^{1,2}$ As estratégias mais comuns utilizam os METs ou relacionamento entre $\mathrm{VO}_{2}$ e FC, permitindo controlar por meio do $\mathrm{VO}_{2}$ a carga de trabalho, volume de treinamento e dispêndio energético da sessão de exercício, a partir de equações metabólicas disponíveis. ${ }^{2}$

Durante muitos anos, a relação entre os diferentes percentuais de $\mathrm{VO}_{2}$ máx (\% $\mathrm{VO}_{2}$ máx $)$ e FCmáx (\%FCmáx) nortearam as recomendações do ACSM, sendo adotadas universalmente. Todavia, o método do \%FCmáx apresenta sérias limitações do ponto de vista prático. Há uma forte tendência em se subestimar a intensidade de exercício em todas as faixas etárias, principalmente em idades mais avançadas. ${ }^{14}$ $\mathrm{O}$ método também é inexato para estimar o $\% \mathrm{VO}_{2}$ máx em exercícios de baixa intensidade. Por exemplo, valores de 40, 50, 60, 80 e $85 \%$ do $\mathrm{VO}_{2}$ máx corresponderiam, respectivamente, a $55,62,70,85$ e $90 \%$ do \%FCmáx. ${ }^{8}$

Outra forma de determinação da intensidade do exercício pela FC é o método da FC de reserva, ou método de Karvonen, ${ }^{15}$ definido como a porcentagem da diferença entre o valor máximo e de repouso (\%FCR). Assume-se que a FC não parte do zero no início do esforço físico, o que permite uma maior correspondência com a intensidade do exercício em termos de $\mathrm{VO}_{2}$, tendo sido inclusive sugerida a substituição do \%FCmáx pelo \%FCR. ${ }^{8}$ Nas últimas recomendações do $\mathrm{ACSM}^{1,8}$ foi proposta, além disso, a utilização do método de reserva pelo $\mathrm{VO}_{2}$ em substituição ao método do $\% \mathrm{VO}_{2}$ máx. Análogo à $\mathrm{FCR}$, esse conceito é definido como a porcentagem da diferença entre o valor máximo e em repouso $\left(\% \mathrm{VO}_{2} \mathrm{R}\right)$. Existe uma premissa que os $\% \mathrm{FCR}$ seriam equivalentes aos $\% \mathrm{VO}_{2} \mathrm{R}$, mas não aos $\% \mathrm{VO}_{2}$ máx, sugerindo uma associação de 1:1 entre $\% \mathrm{FCR}$ e $\% \mathrm{VO}_{2} \mathrm{R}$, em relação exprimida pelas seguintes equações: FCR $=$ (FCmáx FCrepouso $) \%+$ FCrepouso e $\mathrm{VO}_{2} \mathrm{R}=\left(\mathrm{VO}_{2}\right.$ máx - $\mathrm{VO}_{2}$ repouso)\% + $\mathrm{VO}_{2}$ repouso, em que $\mathrm{VO}_{2}$ repouso equivaleria a $1 \mathrm{MET} .{ }^{16}$ Para o ACSM, isso aumentaria as chances de que a intensidade dos exercícios coincidisse com as prescrições baseadas na \%FCR. O \%FCR relacionar-seia melhor ao $\% \mathrm{VO}_{2} \mathrm{R}$ em todos os níveis de aptidão cardiorrespiratória, especialmente em indivíduos com baixa capacidade de exercício e durante as zonas mais baixas de intensidade 
de treinamento. ${ }^{2}$ Por exemplo, para promover saúde, o ACSM recomenda uma intensidade de exercício entre $40 \%-85 \%$ da FCR ou do $\mathrm{VO}_{2} \mathrm{R}$ (ex.: é recomendado uma frequência semanal maior ou igual a 5 dias.sem ${ }^{-1} \mathrm{de}$ exercícios de moderada intensidade; ou maior ou igual a 3 dias.sem ${ }^{-1}$ de exercícios de vigorosa intensidade; ou a combinação de exercícios de moderada a vigorosa intensidade), e um dispêndio energético-alvo de 150 a $400 \mathrm{kcal}$ por sessão de exercício ou 1.000 a $2.000 \mathrm{kcal}$ por semana. O conceito de atividade física de leve intensidade foi definido como $20-39 \% \mathrm{VO}_{2} \mathrm{R}$ FCR ou 1,1-2,9 METs, atividade de moderada intensidade $40 \%-59 \% \mathrm{VO}_{2} \mathrm{R}-\mathrm{FCR}$ ou 3,0-5,9 METs e atividade de vigorosa intensidade $60 \%-85 \% \mathrm{VO}_{2} \mathrm{R}-\mathrm{FCR}$ ou $\geq 6,0$ METs (1 MET correspondendo ao valor de referência de 3,5 $\left.\mathrm{ml} \cdot \mathrm{kg}^{-1} \cdot \mathrm{min}^{-1}\right)^{1}$ (Tabela 1 ).

Em termos práticos, por meio da relação entre $\% \mathrm{FCR}$ e $\% \mathrm{VO}_{2} \mathrm{R}$ estabelece-se um percentual desejado de intensidade dentro da faixa de treinamento desejada, obtendo-se assim valores absolutos para $\mathrm{FC} \mathrm{(bat.min}{ }^{-1}$ ) e $\mathrm{VO}_{2}(\mathrm{ml}$. $\mathrm{kg}^{-1} \cdot \mathrm{min}^{-1}$ ) a serem aplicados durante o exercício. Usualmente, a partir dos valores de $\mathrm{VO}_{2}$ obtidos por meio do cálculo da equação de $\% \mathrm{VO}_{2} \mathrm{R}$ (Gross $\mathrm{VO}_{2}$ ), são calculadas as velocidades de caminhada e corrida ou a potência de pedalagem, expressas pelas seguintes equações, respectivamente: $\mathrm{VO}_{2}$ caminhada $=(0,1 \times \mathrm{V})+$ $(1,8 \times \mathrm{Vx} G)+3,5 ; \mathrm{VO}_{2}$ corrida $=(0,2 \times \mathrm{V})+(0,9$ $\mathrm{x} \mathrm{Vx} \mathrm{G})+3,5 ; \mathrm{VO}_{2}$ cicloergômetro de membros inferiores $=1,8$ (ritmo de trabalho) $/$ peso corporal +7 , em que 3,5 representa o valor de 1 MET ou $\mathrm{VO}_{2}$ repouso em $\mathrm{ml} \cdot \mathrm{kg}^{-1} \cdot \mathrm{min}^{-1}$, $\mathrm{V}$ é a velocidade em $\mathrm{m} \cdot \mathrm{min}^{-1} \mathrm{e}$ G é o grau de inclinação da esteira. ${ }^{2}$ Para cálculo do dispêndio energético das sessões de treinamento, considera-se em primeiro lugar o Net $\mathrm{VO}_{2}$ (Gross $\mathrm{VO}_{2}-\mathrm{VO}_{2}$ de repouso). ${ }^{17}$ Em segundo lugar, é necessário converter o Net $\mathrm{VO}_{2}\left(\mathrm{ml} \cdot \mathrm{kg}^{-1} \cdot \mathrm{min}^{-1}\right)$ para unidade absoluta $\left(1 \cdot \mathrm{min}^{-1}\right)$. Para tal, multiplicase o valor em $\mathrm{ml} \cdot \mathrm{kg}^{-1} \cdot \mathrm{min}^{-1}$ pela massa corporal $(\mathrm{kg})$ e divide-se por 1.000 (isto é, $1.000 \mathrm{ml}$ por litro). Posteriormente, para transformar o $\mathrm{VO}_{2}\left(1 . \mathrm{min}^{-1}\right)$ em dispêndio energético (kcal. $\mathrm{min}^{-1}$ ), multiplica-se o seu valor por 5 (isto é, aproximadamente $5 \mathrm{kcal}$ por litro de $\mathrm{O}_{2}$ durante o exercício em ritmo estável).²

\section{Limitações metodológicas associadas à prescrição da intensidade do exercício aeróbio}

Tabela 1. Classificação da intensidade do exercício com base em atividades contínuas de 20 a 60 minutos de duração.

\begin{tabular}{|c|c|c|c|c|c|c|c|c|c|}
\hline \multirow{3}{*}{ Intensidade } & \multicolumn{5}{|c|}{ Intensidade relativa } & \multicolumn{4}{|c|}{ Intensidade absoluta (MET) por idade } \\
\hline & \multirow{2}{*}{$\begin{array}{l}\% \text { FCR ou } \\
\% \text { VO2R }\end{array}$} & \multirow{2}{*}{ \%FCmáx } & \multirow{2}{*}{$\% \mathrm{VO}_{2}$ máx } & \multicolumn{2}{|c|}{ Percepção de esforço } & \multirow{2}{*}{ METS } & \multirow{2}{*}{$\begin{array}{c}\text { Jovens } \\
\text { (20-39 anos) }\end{array}$} & \multirow{2}{*}{$\begin{array}{l}\text { Meia-idade } \\
\text { (40-64 anos) }\end{array}$} & \multirow{2}{*}{$\begin{array}{c}\text { Idosos } \\
(\geq 65 \text { anos })\end{array}$} \\
\hline & & & & Borg $(6-20)$ & Borg (0-10) & & & & \\
\hline Muito leve & $<30$ & $<57$ & $<37$ & $<9$ & $<1$ & $<2,0$ & $<2,4$ & $\leq 2,0$ & $<1,6$ \\
\hline Leve & $30-39$ & $57-63$ & $37-45$ & $9-11$ & $1-2$ & $2,0-2,9$ & $2,4-4,7$ & $2,0-3,9$ & $1,6-3,1$ \\
\hline Moderada & $40-59$ & $64-76$ & $46-63$ & $12-13$ & $3-4$ & $3,0-5,9$ & $4,8-7,1$ & $4,0-5,9$ & $3,2-4,7$ \\
\hline Vigorosa & $60-89$ & $77-95$ & $64-90$ & $14-17$ & $5-6$ & $6,0-8,7$ & $7,2-10,1$ & $6,0-8,4$ & $4,8-6,7$ \\
\hline Próximo do & & & & & & & & & \\
\hline $\begin{array}{l}\text { máximo - } \\
\text { máximo }\end{array}$ & $\geq 90$ & $\geq 96$ & $\geq 91$ & $\geq 18$ & $\geq 7$ & $\geq 8,8$ & $\geq 10,2$ & $\geq 8,5$ & $\geq 6,8$ \\
\hline
\end{tabular}

$\mathrm{FC}=$ frequência cardíaca; $\mathrm{FCR}$ = frequência cardíaca de reserva; $\mathrm{VO}_{2}=$ consumo de oxigênio; $\mathrm{VO}_{2} \mathrm{R}=$ consumo de oxigênio de reserva; $\mathrm{MET}$ = equivalente metabólico. 


\section{Calculando o dispêndio energético pelo uso dos METs}

Uma importante limitação metodológica na prescrição da intensidade do exercício aeróbio reside no uso dos METs. Na maior parte dos casos assume-se que 1 MET equivaleria a 3,5 $\mathrm{ml} \cdot \mathrm{kg}^{-1} \cdot \mathrm{min}^{-1}$, para um dispêndio energético de $1,0 \mathrm{kcal} \cdot \mathrm{kg}^{-1}$ (massa corporal).h ${ }^{-1}$. Logo, o custo energético das atividades poderia ser expresso como múltiplos da taxa metabólica de repouso (TMR), multiplicando-se a massa corporal $(\mathrm{kg})$ pelo valor do MET e pela duração da atividade [ex.: dispêndio energético $(\mathrm{kcal})=$ (MET da atividade $\mathrm{x}$ massa corporal) $\mathrm{x}$ (duração em $\mathrm{min} / 60 \mathrm{~min}){ }^{1,8} \mathrm{O}$ MET estimado para diferentes atividades e intensidades pode ser obtido pelo Compêndio de atividades físicas proposto por Ainsworth e colaboradores. ${ }^{18} \mathrm{O}$ compêndio é organizado em um sistema de codificação que emprega códigos com cinco dígitos para categorizar as atividades pelo propósito principal ou por grupo principal (dois primeiros dígitos), atividades específicas (últimos três dígitos) e intensidade (dois ou três dígitos na coluna separada).

Contudo, sabe-se que vários fatores podem interferir nessa taxa, como idade, sexo, peso, massa muscular, massa gorda, entre outros. Byrne e colaboradores ${ }^{19}$ mediram a TMR durante 45 minutos, adotando os valores obtidos nos últimos 25 minutos de um período de steady state (estado de equilíbrio), por calorimetria indireta, além do custo energético durante caminhada em esteira rolante $\left(5,6 \mathrm{~km} \cdot \mathrm{h}^{-1}\right)$, em um grupo de 642 mulheres e 127 homens de 18 a 74 anos de idade e massa corporal de 35 a $186 \mathrm{~kg}$. Os valores obtidos para TMR e para o custo energético foram de 2,6 $\pm 0,4(1,61-4,05)$ $\mathrm{ml} \cdot \mathrm{kg}^{-1} \cdot \mathrm{min}^{-1}$ e $0,84 \pm 0,16(0,54-1,35) \mathrm{kcal}$. $\mathrm{kg}^{-1} \cdot \mathrm{h}^{-1}$, respectivamente, sendo menores que os valores de 1 MET geralmente aceitos, de $3,5 \mathrm{ml} \cdot \mathrm{kg}^{-1} \cdot \mathrm{min}^{-1}$ e $1,0 \mathrm{kcal} \cdot \mathrm{kg}^{-1} \cdot \mathrm{h}^{-1}$. Os autores constataram, além disso, que a TMR tinha uma relação inversa com o índice de massa corporal (IMC): quanto menor o IMC, maior era a TMR. Houve, outrossim, influência do sexo e idade sobre a TMR. Recentemente, nosso grupo investigou a TMR em 125 homens saudáveis de 17 a 38 anos de idade e massa corporal de 52,6 a $110,9 \mathrm{~kg} .{ }^{20} \mathrm{O}$ valor médio para a TMR de 3,21 $(2,00-4,40) \mathrm{mL} \cdot \mathrm{kg}^{-1} \cdot \mathrm{min}^{-1}$ foi significativamente menor do que o valor padrão de $3,5 \mathrm{~mL} \cdot \mathrm{kg}^{-1}$. $\min ^{-1}$ (diferença média $=0,29 ; 95 \% \mathrm{CI}=0,20 \mathrm{a}$ $0,37 ; \mathrm{t}=6,7 ; \mathrm{P}<0,001)$.

Grande parte das pesquisas epidemiológicas e ensaios clínicos que analisaram os efeitos da intensidade do exercício (vigorosa intensidade vs. moderada intensidade) sobre parâmetros de saúde cardiovascular e metabólica adotaram intensidades baseadas em múltiplas estimativas do metabolismo de repouso (METs; $1 \mathrm{MET}=3,5$ $\mathrm{ml} \cdot \mathrm{kg}^{-1} \cdot \mathrm{min}^{-1} .^{6}$ Porém, este valor $\left(\mathrm{VO}_{2}\right.$ repouso $=3,5 \mathrm{ml} \cdot \mathrm{kg}^{-1} \cdot \mathrm{min}^{-1}$ ) pode superestimar o valor real medido em alguns indivíduos em até $54 \%$ $\left(1,61 \mathrm{ml} \cdot \mathrm{kg}^{-1} \cdot \mathrm{min}^{-1}\right) \cdot{ }^{19}$ Assim, uma intensidade de prescrição de exercício $\geq 6,0$ METs baseada no valor de 1 MET como sendo de 3,5 ou 1,61 $\mathrm{ml} \cdot \mathrm{kg}^{-1} \cdot \mathrm{min}^{-1}$ corresponderia, respectivamente, a um $\mathrm{VO}_{2}$ de 21,0 e $9,7 \mathrm{ml} \cdot \mathrm{kg}^{-1} \cdot \mathrm{min}^{-1}$, refletindo um erro de superestimativa acima de $50 \%$.

\section{Controle da intensidade e dispêndio energético do treinamento aeróbio pela relação entre $\% \mathrm{FCR}-\% \mathrm{VO}_{2} \mathrm{R}$}

Outro problema importante para a definição da intensidade de exercício é a hipotética relação de 1:1 entre os \%FCR e \% $\mathrm{VO}_{2} \mathrm{R}$. A utilização da FC como um indicador da intensidade metabólica relativa baseia-se em estudos de validação que têm empregado exercícios de curta duração, com aumento progressivo da intensidade. ${ }^{16} \mathrm{Em}$ outras palavras, é ainda incerto se a alteração na FC é um marcador válido de variação da intensidade metabólica relativa durante o exercício prolongado, realizado em diferentes condições ambientais. Neste contexto, Cunha e colaboradores ${ }^{20}$ investigaram o relacionamento entre \%FCR$\% \mathrm{VO}_{2} \mathrm{R}$ e $\% \mathrm{FCR}-\% \mathrm{VO}_{2}$ máx durante sessões prolongadas de corrida realizadas em três 
diferentes intensidades (60\%, 70\% e 80\% $\left.\mathrm{VO}_{2} \mathrm{R}\right) \mathrm{e}$ carga constante de trabalho em ambiente neutro $\left(19^{\circ} \mathrm{C}\right.$ a $\left.22^{\circ} \mathrm{C}\right)$. O hipotético relacionamento de 1:1 entre os \%FCR-\% $\mathrm{VO}_{2} \mathrm{R}$ e $\% \mathrm{FCR}-\% \mathrm{VO}_{2}$ máx não se reproduziu e, em contraste com o que aparentemente ocorre durante teste incremental máximo, ${ }^{21,22} \mathrm{o}$ erro percentual (ex.: dissociação entre os percentuais de FCR e $\mathrm{VO}_{2} \mathrm{R}$ ) entre as variáveis aumentou com a intensidade de exercício. Numa perspectiva prática, esta é uma importante informação para prescrição de exercício, uma vez que estudos prévios têm adotado a estratégia de monitoramento da FC para ajustar a carga de trabalho correspondente à intensidade do exercício desejado e, com isso, garantir que os protocolos de treinamento sejam isocalóricos em adultos saudáveis, ${ }^{23}$ pacientes com insuficiência cardíaca ${ }^{24}$ e adultos obesos. ${ }^{25}$ Em outras palavras, parece que controlar a intensidade do exercício utilizando a FC não seria o suficiente para assegurar que o volume de treinamento pretendido esteja sendo executado, particularmente em intensidades mais elevadas.

Por outro lado, a instabilidade nos relacionamentos entre os $\% \mathrm{FCR}-\% \mathrm{VO}_{2} \mathrm{R}$ e $\% \mathrm{FCR}-\% \mathrm{VO}_{2}$ máx durante o exercício contínuo pode afetar a intensidade de treinamento, especialmente em sessões de intensidade moderada a máxima. É bem-conhecido que, durante exercício prolongado com maior intensidade, pode ocorrer um componente lento da cinética de $\mathrm{VO}_{2}$. Tais aumentos no $\mathrm{VO}_{2}$ têm sido atribuídos a vários mecanismos fisiológicos, incluindo a ativação de grupos musculares adicionais, maior atividade muscular respiratória, recrutamento de fibras musculares do tipo II, aumento da temperatura muscular e níveis mais elevados de lactato sanguíneo, entre outros. ${ }^{26}$ Esses fatores podem, também, influenciar as respostas cardiovasculares durante exercício prolongado. $\mathrm{O}$ aumento no $\mathrm{VO}_{2}$ parece ser concomitante a uma diminuição no volume sistólico e aumento compensatório na FC, com pouca variação do débito cardíaco, o que é conhecido como cardiovascular drift. ${ }^{27}$ Durante o exercício prolongado, o aumento da temperatura corporal e hidratação reduzida podem contribuir para uma diminuição da pressão de enchimento e volume diastólico final, promovendo uma taquicardia ligeiramente maior ${ }^{28} \mathrm{e}$, provavelmente, uma dissociação entre os $\% \mathrm{FCR}$ e $\% \mathrm{VO}_{2} \mathrm{R}$.

Nesse sentido, é importante destacar que o erro percentual na relação entre \%FCR e $\% \mathrm{VO}_{2} \mathrm{R}$ pode ser influenciado pela temperatura ambiente e pelo modo de exercício. Estudos observaram que o aumento no estresse térmico, resultante da elevação na temperatura central e da pele durante o exercício no calor, implica em maior sobrecarga cardiovascular. Desidratação e hipertermia durante o exercício prolongado e intenso podem induzir um declínio no débito cardíaco, fluxo sanguíneo muscular e para a pele, e oferta de oxigênio aos músculos exercitados. ${ }^{29,30}$ Em resumo, quando o exercício no calor é prolongado, o débito cardíaco diminui e a diferença arteriovenosa de oxigênio ampliase ao máximo, ${ }^{31}$ potencialmente limitando o fornecimento de oxigênio para os músculos que estão sendo exercitados. Apenas para ilustrar, Wingo e colaboradores ${ }^{32}$ investigaram o aumento progressivo da FC e a queda do volume de ejeção durante exercício prolongado em nove ciclistas (45 min a 60\% $\mathrm{VO}_{2}$ máx) executado em ambiente quente $\left(35^{\circ} \mathrm{C}\right)$. A FC aumentou $12 \%$ e o volume de ejeção reduziu $16 \%$ durante 45 minutos de exercício, sendo que $\mathrm{VO}_{2}$ aumentou somente cerca de $2 \%$.

$\mathrm{O}$ fato de as respostas fisiológicas a diferentes modos de exercício serem diferentes é bem conhecido. Nassis e Geladas ${ }^{33}$ compararam as respostas cardiovasculares durante sessões prolongadas de ciclismo e corrida de 90 minutos a $60 \% \mathrm{VO}_{2}$ máx em condições termoneutras $(23,8$ $\pm 0,3{ }^{\circ} \mathrm{C}$ ), concluindo que o cardiovascular drift seria mais pronunciado no ciclismo do que na corrida, apesar do maior estresse térmico, desidratação e aumento da FC no último tipo de exercício. Isto sugere que a massa muscular ativa desempenhou um papel determinante nas respostas cardiovasculares. Porém, o efeito do modo de exercício sobre o cardiovascular drift 
durante exercício prolongado em ambiente quente ainda não foi definido. Em outras palavras, o cardiovascular drift observado durante sessões prolongadas de exercício em condições ambientais quentes pode ser agravado pelo modo de exercício, potencializando ainda mais a dissociação entre $\% \mathrm{FCR}$ e $\% \mathrm{VO}_{2} \mathrm{R}$, e, por conseguinte, aumentando o erro na estimativa do dispêndio energético.

Enfim, outra questão a ser levada em consideração é o quanto as cargas de trabalho definidas pelas equações metabólicas do ACSM reproduziriam o $\mathrm{VO}_{2} \mathrm{R}$ para uma dada intensidade, que estaria similarmente associada à FCR e, consequentemente, ao gasto calórico almejado. De fato, não se sabe ao certo o quanto as velocidades ou potências definidas por tais equações reproduzem o $\% \mathrm{VO}_{2} \mathrm{R}$ com que se deseja trabalhar. Por exemplo, foi possível localizar apenas dois estudos, todos de nosso grupo, abordando esta questão em ambiente neutro, ${ }^{20,34}$ concluindo que a equação metabólica de corrida do ACSM reproduziu o valor predito de FCR, mas superestimou o $\mathrm{VO}_{2}$. Numa perspectiva prática, seria útil determinar se as equações metabólicas de caminhada e ciclismo do ACSM reproduzem com precisão o dispêndio energético alvo de sessões isocalóricas de exercício aeróbio, realizadas com diferentes intensidades e condições ambientais.

\section{Treinamento de alta}

intensidade e curta duração

vs. moderada intensidade

\section{e longa duração: qual é a}

melhor estratégia para o emagrecimento?

O metabolismo energético constitui um processo complexo e não parece se resumir a uma simples contabilização de quantas calorias se ingere em relação a quantas calorias se gasta. É sabido que a dieta alimentar parece ser a melhor estratégia para perder ou ganhar massa corporal; por outro lado, evidências apontam para o papel crucial do exercício físico, principalmente o exercício aeróbio, para um emagrecimento saudável, ou seja, diminuição de gordura com manutenção da massa dela isenta. ${ }^{35}$

Por outrolado, no tocante ao emagrecimento, outros fatores para além do gasto energético decorrente do exercício aeróbio devem ser considerados. Muitos estudiosos têm proposto questionamentos, por exemplo: até que ponto o balanço energético negativo gerado pelo exercício físico é eficaz para o emagrecimento, já que alguns estudos apontam para um aumento da ingestão alimentar após exercício físico, contrabalanceando este deficit energético ${ }^{36}$ Com o intuito de responder a essa dúvida, Whybrow e colaboradores ${ }^{38}$ investigaram os efeitos agudos de diferentes intensidades de exercício sobre o apetite, ingestão alimentar, dispêndio e balanço energético em 12 sujeitos (seis mulheres) que realizaram os seguintes exercícios em ordem randomizada: a) sessão controle (nenhum exercício adicionado à rotina habitual de tarefas da vida diária); b) duas sessões de exercício por dia de 40 minutos de moderada intensidade com o intuito de atingir um gasto-alvo de $28,6 \mathrm{~kJ} / \mathrm{kg}$ de massa corporal; e c) três sessões de exercício por dia de 40 minutos de alta intensidade com o intuito de atingir um dispêndio de $57,1 \mathrm{~kJ} / \mathrm{kg}$ de massa corporal. Constatou-se que o dispêndio energético gerado pela prática de exercício começa a ser compensado ou contrabalanceado pela ingestão alimentar num curso de uma a duas semanas, tanto em homens quanto em mulheres normoponderais.

Mais recentemente, King e colaboradores ${ }^{39}$ relataram que o efeito do exercício sobre a regulação do apetite envolveria, pelo menos, dois processos: 1) aumento em todos os sinais orexígenos; 2) um concomitante aumento na eficiência e interpretação de sinais de saciedade em refeições fixas. No entanto, os mesmos autores alegam que esses processos não operam com a mesma força e eficácia em todos os praticantes de exercícios. Juntas, as forças dos dois processos podem determinar se indivíduos 
perderão peso com a prática de exercício ou se o peso corporal será mantido através do efeito compensatório do aumento da ingestão alimentar após a sessão de exercício.

Em relação às respostas compensatórias ao deficit energético gerado pelo exercício, King e colaboradores ${ }^{39}$ sugeriram que a influência das respostas compensatórias metabólicas (respostas automáticas ou obrigatórias, como a TMR e o dispêndio energético gerado por outras atividades fisiológicas) e comportamentais (respostas voluntárias ou facultativas, como a ingestão alimentar), seria um ponto-chave no tocante à barreira para perda de peso induzida pela prática de atividades físicas. As respostas compensatórias comportamentais teriam maior influência sobre o balanço energético do que sobre as respostas metabólicas. Portanto, as contribuições da atividade física sobre o balanço energético seriam menos expressivas do que a contribuição gerada pela ingestão alimentar. ${ }^{40}$ Em suma, parece que o efeito de sessões agudas de atividades físicas depende extremamente da intensidade empregada na atividade. Exercícios de moderada a máxima intensidades ( $>$ $60 \% \mathrm{VO}_{2}$ máx) parecem ser mais eficazes no tocante ao efeito supressor sobre biomarcadores relacionados à ingestão alimentar. ${ }^{41}$

Nesta mesma ótica, vários estudos especularam que exercícios de baixa intensidade e curta duração parecem não induzir efeito supressor sobre a fome. Somente exercícios de alta intensidade e longa duração teriam efeito sobre a ingestão alimentar, comparados a exercícios de curta duração. Assim, exercícios extenuantes parecem suprimir o apetite e induzir um efeito denominado anorexia induzida pelo exercício. ${ }^{38}$ Esses efeitos supressores parecem estar ligados a mecanismos de sinalização de adipocitocinas relacionadas à saciedade, como por a leptina e adiponectina. Ambas somente sofreriam alterações em suas concentrações após sessões de exercícios associadas a um gasto energético superior a $800 \mathrm{kcal}$.

Além disso, exercícios de alta intensidade favoreceriam mais a oxidação lipídica e o balanço energético negativo do que exercícios de intensidade baixa ou moderada. ${ }^{7}$ Somado a isso, haveria uma maior diminuição na gordura abdominal subcutânea e visceral como resultado de treinamento de maior intensidade..$^{10,12}$

Com base nisso, pode-se pensar que se o exercício físico tem papel determinante sobre o controle do apetite e composição corporal, tais efeitos parecem ser amplificados pela magnitude da sua intensidade. Para além desses benefícios, exercícios de intensidades vigorosas promoveriam melhorias adicionais na tolerância à glicose e aptidão cardiorrespiratória, quando comparados a exercícios de baixa intensidade. ${ }^{11}$

Para a correta estimativa do dispêndio energético devido à atividade física, também é preciso considerar o período pós-exercício. A TMR é o maior componente do gasto energético diário, responsável por $50 \%$ (ex.: indivíduos fisicamente ativos) a 70\% (ex.: indivíduos sedentários) do dispêndio total. ${ }^{42}$ Imediatamente após o exercício, esse metabolismo permanece elevado por vários minutos e até por horas. ${ }^{43} \mathrm{O}$ termo EPOC (excess post-exercise oxygen consumption - ou excesso de consumo de oxigênio pósexercício) tem sido adotado para designar esse incremento no metabolismo. ${ }^{44}$ Diversas variáveis são apontadas como determinantes do EPOC, dentre elas o aumento da temperatura corporal, níveis de catecolaminas, tiroxinas, glicocorticoides, metabolismo de ácidos graxos, reabastecimento dos estoques de oxihemoglobina e oximioglobina, restauração dos fosfagênios e energia necessária para reconversão do lactato em glicogênio, hiperemia e ventilação elevada, presença de cortisol e lesão muscular induzida pelo treinamento. ${ }^{43-45}$

A magnitude e duração do EPOC também são influenciadas pela intensidade e duração

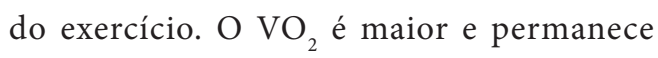
elevado durante um período mais longo após o exercício de alta intensidade, em comparação ao exercício de intensidade baixa a moderada. ${ }^{43-45}$ De fato, tanto a intensidade quanto a duração do exercício aeróbio implicam em EPOC elevado; 
a diferença é que o aumento do EPOC em relação à duração do exercício ocorre de forma linear, enquanto seu aumento se dá de forma exponencial em relação à intensidade. ${ }^{43-45} \mathrm{Em}$ artigo de revisão, LaForgia e colaboradores ${ }^{46}$ investigaram os efeitos da intensidade e duração do exercício sobre o EPOC, concluindo que o mesmo estaria relacionado prioritariamente à variável intensidade.

Partindo-se do princípio de que é possível permanecer mais minutos em exercício com intensidade alta quando este é realizado de forma intervalada, em comparação com a contínua, LaForgia e colaboradores ${ }^{46}$ investigaram o EPOC obtido em sessões contínuas (30 min a $70 \% \mathrm{VO}_{2}$ máx) e intervalada (20 séries de 1 minuto a $105 \% \mathrm{VO}_{2}$ máx com 2 min de recuperação), ambas com o mesmo volume total, em oito corredores de meia distância (idade $=21 \pm 3$ anos; massa corporal $=67,8 \pm$ $5,1 \mathrm{~kg} ; \mathrm{VO}_{2}$ máx $\left.=69,2 \pm 4,0 \mathrm{ml} \cdot \mathrm{kg}^{-1} \cdot \mathrm{min}^{-1}\right)$. Após 9 horas de recuperação, as sessões intervalada e contínua resultaram, respectivamente, num EPOC de 15,0 $\pm 3,3$ e 6,9 $\pm 3,8$ litros, sendo ambas significativamente maiores que a sessão controle $(\mathrm{P}<0,001)$. Esses valores representaram 13,8\% e 7,1\% do dispêndio energético total líquido de ambos os protocolos de treinamento. Concluiuse que o EPOC foi significativamente maior na sessão intervalada supramáxima, quando comparada à sessão submáxima contínua com a mesma quantidade total de trabalho realizado. Portanto, essa estratégia pode ser interessante, já que pacientes sedentários podem exercitar-se por tempo menor a uma intensidade que produza um EPOC maior. Além disso, indivíduos sedentários possuem baixa tolerância a esforços muito prolongados (ex.: atividades contínuas com 30 a 60 min de duração). Consequentemente, sessões intervaladas com breves períodos de estímulo em alta intensidade, alternando com maiores períodos de recuperação em baixa intensidade, podem cronicamente resultar em melhores benefícios para aptidão cardiorrespiratória e composição corporal do que sessões contínuas de moderada intensidade e longa duração.

Em recente revisão sobre o assunto, Kessler e colaboradores ${ }^{9}$ analisaram os estudos que investigaram os efeitos do treinamento intervalado de alta intensidade (high intensity training - HIT) sobre os marcadores de risco cardiovasculares e metabólicos, com aqueles obtidos em sessões contínuas de treinamento de moderada intensidade e longa duração. Basicamente, os autores descreveram dois tipos de HIT, usualmente adotados por estudos com esteira rolante (ex.: corrida e caminhada) e cicloergômetro: 1) Sprint Interval Training (SIT), caracterizado por 4-6 tiros de 30 segundos seguidos por 4-5 minutos de recuperação (a maior parte dos estudos foi conduzida em ciclo ergômetro); 2) Aerobic Interval Training (AIT), tipicamente realizado em menor intensidade que o SIT, mas com maiores períodos de tempo. Os estudos analisados nesta revisão, que adotaram o AIT, aplicaram 4-6 séries de 4 minutos de estímulo em intensidade vigorosa (80-95\% $\mathrm{VO}_{2}$ máx), seguidos por 3-4 minutos de recuperação, na esteira ou cicloergômetro. $\mathrm{Na}$ opinião dos autores, contrariamente ao SIT, a abordagem do AIT tem sido mais utilizada em jovens saudáveis e grupos de risco (ex.: idosos, pacientes com coronariopatia, etc.). Quanto aos resultados obtidos, ambas as abordagens de HIT seriam efetivas para o aprimoramento da aptidão cardiorrespiratória e melhora da sensibilidade à insulina, com resultados similares ou superiores ao treinamento contínuo prolongado de moderada intensidade.

Em relação aos resultados associados à melhoria da composição corporal em pacientes com sobrepeso ou obesidade (ex.: decréscimos no IMC, percentual de gordura e circunferência de cintura), os autores observaram que tais resultados levariam, pelo menos, 12 semanas para serem atingidos de forma significativa. Por outro lado, os autores relataram que apesar de ser mais vantajoso para os marcadores de risco à saúde do que o treinamento contínuo, o HIT pode não ser o método mais apropriado para todos. Nesse sentido, foram destacados 
os seguintes aspectos: a) necessidade de uma estrutura adequada para supervisão das sessões de treinamento (especialmente em pacientes sedentários); b) liberação médica devido à natureza do exercício de alta intensidade; c) em função do alto impacto do exercício, a corrida em esteira pode não ser uma modalidade apropriada de exercício para pacientes com problemas ou riscos ortopédicos (nesse caso, tanto a caminhada com inclinação como o cicloergômetro podem oferecer os mesmos benefícios sem risco de lesão); d) o HIT requer elevado grau de motivação para alcançar a intensidade desejada (logo, este pode não ser o método preferido de treinamento para a maioria das pessoas).

\section{Conclusão}

Há evidências amplamente favoráveis à prescrição de exercícios de alta intensidade e curta duração para promover melhores mudanças sobre a aptidão cardiorrespiratória, composição corporal e fatores de risco cardiovasculares, em comparação às sessões de moderada intensidade e longa duração. No entanto, deve-se notar que uma boa prescrição de exercícios deveria considerar aspectos comportamentais, buscando adesão em longo prazo e atendimento aos anseios do praticante. Em outras palavras, a melhor combinação das variáveis de prescrição do treinamento aeróbio será aquela que resultará na permanência e satisfação do indivíduo no programa de exercício. Nesse sentido, até onde foi o presente esforço de revisão, programas de emagrecimento deveriam propor rotinas de exercícios no qual o dispêndio energético fosse o maior possível, com a maior intensidade que se possa aplicar. Evidentemente, isso dependerá da aceitação do programa por parte do praticante - optar por exercícios de intensidade e volume menores é sempre válido, com o propósito de manter a adesão ao programa proposto.

\section{Referências}

1. Garber CE, Blissmer B, Deschenes MR,
Franklin BA, Lamonte MJ, Lee IM, et al. American College of Sports Medicine position stand. Quantity and quality of exercise for developing and maintaining cardiorespiratory, musculoskeletal, and neuromotor fitness in apparently healthy adults: guidance for prescribing exercise. Med Sci Sports Exerc. 2011 Jul;43(7):1334-59. http:// dx.doi.org/10.1249/MSS.0b013e318213fefb.

2. ACSM. ACSM's guidelines for exercise testing and prescription. 8th ed. Baltimore: Lippincott Williams \& Wilkins; 2009.

3. CDC. Self-reported physical inactivity by degree of urbanization--United States, 1996. MMWR Morb Mortal Wkly Rep. 1998 Dec 25;47(50):1097-100.

4. Ogden CL, Carroll MD, Curtin LR, McDowell MA, Tabak CJ, Flegal KM. Prevalence of overweight and obesity in the United States, 1999-2004. JAMA. 2006 Apr 5;295(13):1549-55. http://dx.doi.org/10.1001/jama.295.13.1549.

5. Kesaniemi YK, Danforth E, Jr., Jensen MD, Kopelman PG, Lefebvre P, Reeder BA. Doseresponse issues concerning physical activity and health: an evidence-based symposium. Med Sci Sports Exerc. 2001 Jun;33(6 Suppl):S351-8.

6. Swain DP, Franklin BA. Comparison of cardioprotective benefits of vigorous versus moderate intensity aerobic exercise. Am J Cardiol. 2006 Jan 1;97(1):141-7. http://dx.doi. org/10.1016/j.amjcard.2005.07.130.

7. Tremblay A, Simoneau JA, Bouchard C. Impact of exercise intensity on body fatness and skeletal muscle metabolism. Metabolism. 1994 Jul;43(7):814-8. http://dx.doi.org/00260495(94)90259-3.

8. ACSM. American College of Sports Medicine Position Stand. The recommended quantity and quality of exercise for developing and maintaining cardiorespiratory and muscular fitness, and flexibility in healthy adults. Med Sci Sports Exerc. 1998 Jun;30(6):975-91.

9. Kessler HS, Sisson SB, Short KR. The potential for high-intensity interval training to reduce cardiometabolic disease risk. Sports Med. 2012 Jun 1;42(6):489-509. http://dx.doi. org/10.2165/11630910-000000000-00000.

10. Irving BA, Davis CK, Brock DW, Weltman JY, Swift D, Barrett EJ, et al. Effect of exercise training intensity on abdominal visceral fat and body composition. Med Sci Sports Exerc. 2008 Nov;40(11):1863-72. http://dx.doi. org/10.1249/MSS.0b013e3181801d40.

11. Nybo L, Sundstrup E, Jakobsen MD, Mohr M, Hornstrup T, Simonsen L, et al. Highintensity training versus traditional exercise 
interventions for promoting health. Med Sci Sports Exerc. 2010 Oct;42(10):1951-8. http:// dx.doi.org/10.1249/MSS.0b013e3181d99203..

12. Trilk JL, Singhal A, Bigelman KA, Cureton KJ. Effect of sprint interval training on circulatory function during exercise in sedentary, overweight/obese women. Eur J Appl Physiol. 2011 Aug;111(8):1591-7. http://dx.doi. org/10.1007/s00421-010-1777-z.

13. Lee JY, Jensen BE, Oberman A, Fletcher GF, Fletcher BJ, Raczynski JM. Adherence in the training levels comparison trial. Med Sci Sports Exerc. 1996 Jan;28(1):47-52.

14. Whaley MH, Kaminsky LA, Dwyer GB, Getchell LH, Norton JA. Predictors of overand underachievement of age-predicted maximal heart rate. Med Sci Sports Exerc. 1992 Oct;24(10):1173-9.

15. Karvonen MJ, Kentala E, Mustala O. The effects of training on heart rate; a longitudinal study. Ann Med Exp Biol Fenn. 1957;35(3):307-15.

16. Cunha FA, Farinatti PT, Midgley AW. Methodological and practical application issues in exercise prescription using the heart rate reserve and oxygen uptake reserve methods. J Sci Med Sport. 2011 Jan;14(1):46-57. http://dx.doi.org/10.1016/j. jsams.2010.07.008.

17. Swain DP. Energy cost calculations for exercise prescription: an update. Sports Med. 2000 Jul;30(1):17-22.

18. Ainsworth BE, Haskell WL, Herrmann SD, Meckes N, Bassett DR, Jr., Tudor-Locke C, et al. 2011 Compendium of Physical Activities: a second update of codes and MET values. Med Sci Sports Exerc. Aug;43(8):1575-81. http:// dx.doi.org/10.1249/MSS.0b013e31821ece12.

19. Byrne NM, Hills AP, Hunter GR, Weinsier RL, Schutz Y. Metabolic equivalent: one size does not fit all. J Appl Physiol. 2005 Sep;99(3):1112-9. http://dx.doi.org/10.1152/ japplphysiol.00023.2004.

20. Cunha FA, Midgley AW, Montenegro R, Oliveira RB, Farinatti PT. Metabolic equivalent concept in apparently healthy men: a re-examination of the standard oxygen uptake value of $3.5 \mathrm{~mL} . \mathrm{kg}-1 . \mathrm{min}-1$. Appl Physiol Nutr Metab. 2013 Nov;38(11):11159. http://dx.doi.org/10.1139/apnm-20120492:10.1139/apnm-2012-0492.

21. Cunha FA, Midgley AW, Monteiro WD, Campos FK, Farinatti PT. The relationship between oxygen uptake reserve and heart rate reserve is affected by intensity and duration during aerobic exercise at constant work rate. Appl Physiol Nutr Metab. 2011
Dec;36(6):839-47. http://dx.doi.org/10.1139/ h11-100:10.1139/h11-100.

22. Swain DP, Leutholtz BC. Heart rate reserve is equivalent to \%VO2 reserve, not to \%VO2max. Med Sci Sports Exerc. 1997 Mar;29(3):410-4.

23. Swain DP, Leutholtz BC, King ME, Haas LA, Branch JD. Relationship between \% heart rate reserve and \% VO2 reserve in treadmill exercise. Med Sci Sports Exerc. 1998 Feb;30(2):318-21.

24. Gormley SE, Swain DP, High R, Spina RJ, Dowling EA, Kotipalli US, et al. Effect of intensity of aerobic training on VO2max. Med Sci Sports Exerc. 2008 Jul;40(7):1336-43. http:// dx.doi.org/10.1249/MSS.0b013e31816c4839.

25. Wisloff U, Stoylen A, Loennechen JP, Bruvold M, Rognmo O, Haram PM, et al. Superior cardiovascular effect of aerobic interval training versus moderate continuous training in heart failure patients: a randomized study. Circulation. 2007 Jun 19;115(24):3086-94. http://dx.doi.org/10.1161/ CIRCULATIONAHA.106.675041.

26. Schjerve IE, Tyldum GA, Tjonna AE, Stolen $\mathrm{T}$, Loennechen JP, Hansen HE, et al. Both aerobic endurance and strength training programmes improve cardiovascular health in obese adults. Clin Sci (Lond). 2008 Nov;115(9):283-93. http://dx.doi.org/10.1042/ CS20070332:CS20070332.

27. Jones AM, Grassi B, Christensen PM, Krustrup P, Bangsbo J, Poole DC. Slow component of V.O kinetics: mechanistic bases and practical applications. Med Sci Sports Exerc. 2011 Nov;43(11):2046-62. http://dx.doi. org/10.1249/MSS.0b013e31821fcfc1.

28. Coyle EF, Gonzalez-Alonso J. Cardiovascular drift during prolonged exercise: new perspectives. Exerc Sport Sci Rev. 2001 Apr;29(2):88-92.

29. Fritzsche RG, Switzer TW, Hodgkinson BJ, Coyle EF. Stroke volume decline during prolonged exercise is influenced by the increase in heart rate. J Appl Physiol. 1999 Mar;86(3):799-805.

30. Gonzalez-Alonso J, Crandall CG, Johnson JM. The cardiovascular challenge of exercising in the heat. J Physiol. 2008 Jan 1;586(1):45-53. http://dx.doi.org/10.1113/ jphysiol.2007.142158.

31. Nybo L, Nielsen B. Hyperthermia and central fatigue during prolonged exercise in humans. J Appl Physiol. 2001 Sep;91(3):1055-60.

32. Rowell LB. Human cardiovascular adjustments to exercise and thermal stress. Physiol Rev. 1974 Jan;54(1):75-159. 
33. Wingo JE, Lafrenz AJ, Ganio MS, Edwards GL, Cureton KJ. Cardiovascular drift is related to reduced maximal oxygen uptake during heat stress. Med Sci Sports Exerc. 2005 Feb;37(2):248-55. http://dx.doi.org/10.1249/01. MSS.0000152731.33450.95.

34. Nassis GP, Geladas ND. Cardiac output decline in prolonged dynamic exercise is affected by the exercise mode. Pflugers Arch. 2002 Dec;445(3):398-404. 10.1007/s00424-0020935-5:10.1007/s00424-002-0935-5.

35. Cunha FA, Catalao RP, Midgley AW, Gurgel J, Porto F, Farinatti PT. Do the speeds defined by the American College of Sports Medicine metabolic equation for running produce target energy expenditures during isocaloric exercise bouts? Eur J Appl Physiol. 2012 Aug;112(8):3019-26. http://dx.doi.org/10.1007/ s00421-011-2275-7.

36. Jakicic JM, Otto AD. Physical activity considerations for the treatment and prevention of obesity. Am J Clin Nutr. 2005 Jul;82(1 Suppl):226S-9S. 82/1/226S [pii]:82/1/226S [pii].

37. Saris WH. The role of exercise in the dietary treatment of obesity. Int J Obes Relat Metab Disord. 1993 Feb;17 Suppl 1:S17-21.

38. Whybrow S, Hughes DA, Ritz P, Johnstone AM, Horgan GW, King N, et al. The effect of an incremental increase in exercise on appetite, eating behaviour and energy balance in lean men and women feeding ad libitum. Br J Nutr. 2008 Nov;100(5):1109-15. http://dx.doi.org/10.1017/S0007114508968240.

39. King NA, Caudwell PP, Hopkins M, Stubbs JR, Naslund E, Blundell JE. Dualprocess action of exercise on appetite control: increase in orexigenic drive but improvement in meal-induced satiety. Am J Clin Nutr. 2009 Oct;90(4):921-7. http://dx.doi. org/10.3945ajcn.2009.27706.

40. King NA, Hester J, Gately PJ. The effect of a medium-term activity- and diet-induced energy deficit on subjective appetite sensations in obese children. Int J Obes (Lond). 2007 Feb;31(2):334-9. http://dx.doi. org/10.1038/sj.ijo.0803391.

41. Blundell JE, Finlayson G. Is susceptibility to weight gain characterized by homeostatic or hedonic risk factors for overconsumption? Physiol Behav. 2004 Aug;82(1):21-5. http:// dx.doi.org/10.1016/j.physbeh.2004.04.021.

42. Bouassida A, Chamari K, Zaouali M, Feki Y, Zbidi A, Tabka Z. Review on leptin and adiponectin responses and adaptations to acute and chronic exercise. Br J Sports Med. 2010 Jul;44(9):620-30. http://dx.doi. org/10.1136/bjsm.2008.046151.

43. Clark HD, Hoffer LJ. Reappraisal of the resting metabolic rate of normal young men. Am J Clin Nutr. 1991 Jan;53(1):21-6.

44. Gaesser GA, Brooks GA. Metabolic bases of excess post-exercise oxygen consumption: a review. Med Sci Sports Exerc. 1984;16(1):29-43

45. Borsheim E, Bahr R. Effect of exercise intensity, duration and mode on postexercise oxygen consumption. Sports Med. 2003;33(14):1037-60. http://dx.doi. org/10.2165/00007256-200333140-00002.

46. LaForgia J, Withers RT, Gore CJ. Effects of exercise intensity and duration on the excess post-exercise oxygen consumption. J Sports Sci. 2006 Dec;24(12):1247-64. http://dx.doi. org/10.1080/02640410600552064.

47. Laforgia J, Withers RT, Shipp NJ, Gore CJ. Comparison of energy expenditure elevations after submaximal and supramaximal running. J Appl Physiol. 1997 Feb;82(2):661-6. 


\section{Autores}

\section{Ada Fernanda P. S. Lima}

Laboratório de Atividade Física e Promoção da Saúde. Instituto de Educação Física e Desportos. Universidade do Estado do Rio de Janeiro. Rio de Janeiro, RJ, Brasil.

\section{Alexandre H. Okano}

Departamento de Educação Física. Centro de Ciências da Saúde. Universidade Federal do Rio Grande do Norte. Natal, RN, Brasil.

\section{Alice R. Sampaio}

Laboratório de Atividade Física e Promoção da Saúde. Instituto de Educação Física e Desportos. Universidade do Estado do Rio de Janeiro. Rio de Janeiro, RJ, Brasil.

\section{Ana Paula M. Guttierres}

Laboratório de Atividade Física e Promoção da Saúde. Instituto de Educação Física e Desportos. Universidade do Estado do Rio de Janeiro. Rio de Janeiro, RJ, Brasil.

\section{Astrogildo V. Oliveira Júnior}

Departamento de Educação Física e Folclore. Colégio Pedro II. Rio de Janeiro, RJ, Brasil.

\section{Brenno S. Silva}

Programa de Pós-graduação em Ciências da Atividade Física. Universidade Salgado de Oliveira. Niterói, RJ, Brasil.

\section{Daniel A. Bottino}

Laboratório de Pesquisas Clínicas e Experimentais em Biologia Vascular. Centro Biomédico.

Universidade do Estado do Rio de Janeiro. Rio de Janeiro, RJ, Brasil.

\section{Dionizio Mendes Ramos Filho}

Laboratório de Bioenergética e Fisiologia Mitocondrial. Centro de Ciências da Saúde. Universidade Federal do Rio de Janeiro. Rio de Janeiro, RJ, Brasil.

\section{Elirez B. Silva}

Laboratório de Pesquisa Clínica Escola. Departamento de Fisioterapia. Universidade Gama Filho. Rio de Janeiro, RJ, Brasil.

\section{Ercole C. Rubini}

Laboratório de Fisiologia do Exercício. Universidade Estácio de Sá. Rio de Janeiro, RJ, Brasil.

\section{Fabrício V. A. Vasconcellos}

Centro de Investigação, Formação, Inovação, Intervenção e Desporto. Faculdade de Desporto. Universidade do Porto. Porto, Portugal.

\section{Felipe A. da Cunha}

Programa de Pós-graduação em Ciências Médicas. Universidade do Estado do Rio de Janeiro. Rio de Janeiro, RJ, Brasil.

\section{Fernanda Monteiro}

Laboratório de Atividade Física e Promoção da Saúde. Instituto de Educação Física e Desportos. Universidade do Estado do Rio de Janeiro. Rio de Janeiro, RJ, Brasil.

\section{Flávia Porto}

Programa de Pós-graduação em Ciências do Exercício e do Esporte. Universidade Gama Filho. Rio de Janeiro, RJ, Brasil. 


\section{Gustavo C. Lopes}

Laboratório de Atividade Física e Promoção da Saúde. Instituto de Educação Física e Desportos. Universidade do Estado do Rio de Janeiro. Rio de Janeiro, RJ, Brasil.

\section{Jonas L. Gurgel}

Programa de Pós-graduação em Ciências

Cardiovasculares. Universidade Federal Fluminense.

Niterói, RJ, Brasil.

\section{Jonathan Myers}

VA Palo Alto Health Care System. Cardiology Division. Stanford University. Palo Alto, California, United States.

\section{Karynne Grutter}

Laboratório de Anatomia Humana. Universidade Castelo Branco. Rio de Janeiro, RJ, Brasil.

\section{Lenifran Matos-Santos}

Programa de Pós-graduação em Ciências da Atividade Física. Universidade Salgado de Oliveira. Niterói, RJ, Brasil.

\section{Lorena Paes}

Programa de Pós-graduação em Fisiopatologia Clínica e Experimental. Universidade do Estado do Rio de Janeiro. Rio de Janeiro, RJ, Brasil.

\section{Luciane P. da Costa}

Laboratório de Atividade Física e Promoção da Saúde. Instituto de Educação Física e Desportos. Universidade do Estado do Rio de Janeiro. Rio de Janeiro, RJ, Brasil.

\section{Luiz G. Kraemer-Aguiar}

Departamento de Medicina Interna. Faculdade de Ciências Médicas. Universidade do Estado do Rio de Janeiro. Rio de Janeiro, RJ, Brasil.

\section{Matheus R. Hausen}

Laboratório de Atividade Física e Promoção da Saúde. Instituto de Educação Física e Desportos. Universidade do Estado do Rio de Janeiro. Rio de Janeiro, RJ, Brasil.

\section{Paulo T. V. Farinatti}

Laboratório de Atividade Física e Promoção da Saúde. Instituto de Educação Física e Desportos. Universidade do Estado do Rio de Janeiro. Rio de Janeiro, RJ, Brasil.

\section{Rafael A. Montenegro}

Programa de Pós-graduação em Fisiopatologia Clínica e Experimental. Universidade do Estado do Rio de Janeiro. Rio de Janeiro, RJ, Brasil.

\section{Raul A. Freire}

Programa de Pós-graduação em Ciências da Atividade Física. Universidade Salgado de Oliveira. Niterói, RJ, Brasil.

\section{Renato O. Massaferri}

Programa de Pós-graduação em Ciências da Atividade Física. Universidade Salgado de Oliveira. Niterói, RJ, Brasil.

\section{Ricardo B. Oliveira}

Laboratório de Atividade Física e Promoção da Saúde. Instituto de Educação Física e Desportos. Universidade do Estado do Rio de Janeiro. Rio de Janeiro, RJ, Brasil.

\section{Ricardo G. Cordeiro}

Programa de Pós-graduação em Ciências da Atividade Física. Universidade Salgado de Oliveira. Niterói, RJ, Brasil.

\section{Sérgio Machado}

Programa de Pós-graduação em Ciências da Atividade Física. Universidade Salgado de Oliveira. Niterói, RJ, Brasil.

\section{Taciana Pinto}

Laboratório de Atividade Física e Promoção da Saúde. Instituto de Educação Física e Desportos. Universidade do Estado do Rio de Janeiro. Rio de Janeiro, RJ, Brasil.

\section{Tainah P. Lima Monteiro}

Programa de Pós-graduação em Ciências Médicas. Faculdade de Ciências Médicas. Universidade do Estado do Rio de Janeiro. Rio de Janeiro, RJ, Brasil.

\section{Tânia M. P. F. Paschoalino}

Hospital Universitário Antônio Pedro. Universidade Federal Fluminense. Niterói, RJ, Brasil.

\section{Walace D. Monteiro}

Laboratório de Atividade Física e Promoção da Saúde. Instituto de Educação Física e Desportos. Universidade do Estado do Rio de Janeiro. Rio de Janeiro, RJ, Brasil. 